\title{
Simulation of the laser acceleration experiment at the Fermilab/NICADD photoinjector laboratory*
}

\author{
P. Piot, Fermi National Accelerator Laboratory, Batavia, IL 60510, USA, \\ R. Tikhoplav, A.C. Melissinos, University of Rochester, Rochester NY 14627, USA.
}

\section{Abstract}

The possibility of using laser beam to accelerate electrons in a waveguide structure with dimension much larger than the laser wavelength was proposed by Pantel and analytically investigated by Xie. In the present paper we present the status of our experimental plan to demonstrate the laser/e $\mathrm{e}^{-}$interaction using an $\mathrm{e}^{-}$beam with initial energy of 40-50 MeV.

\section{INTRODUCTION}

Laser acceleration of electrons relies on using the e.m. field associated with the propagating laser beam to accelerate electrons. Several schemes have been proposed based on inverse radiation mechanisms that can provide a longitudinal accelerating field. In our case, we plan to copropagate an $\mathrm{e}^{-}$beam and a radially polarized laser within a structure that provides weak focusing for the laser (see schematics in Fig. 1).

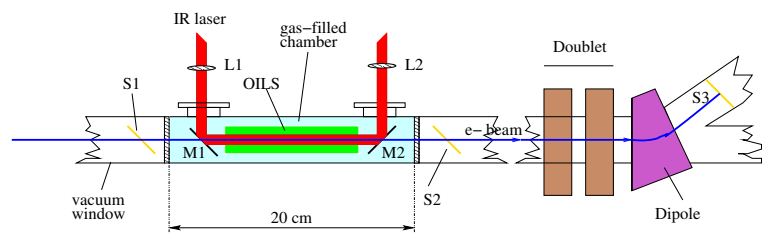

Figure 1: The laser beam enters from the top left. After propagating passing through a lens (L1) and reflecting from the apertured mirror (M1), the beam co-propagates with the $\mathrm{e}^{-}$beam through the open iris-loaded structure (OILS) [green rectangle]. The laser beam is then extracted from the chamber thanks to a second apertured mirror (M2) and then transported to a diagnostics station.

In virtue of the Lawson-Woodward theorem [1], the laser- $\mathrm{e}^{-}$beam interaction must be limited, and there must exist an inverse process that make the $\mathrm{e}^{-}$to radiate. Because it will be necessary to fill the interaction region with gas (see below) this process corresponds Cerenkov radiation.

\section{THE OPEN IRIS-LOADED STRUCTURE CONCEPT}

The acceleration of $\mathrm{e}^{-}$by coupling the laser using an open iris-loaded structure (OILS) was proposed by Pan-

\footnotetext{
${ }^{*}$ work supported by URA under contract No. DE-AC02-76CH00300 with US-DOE.
}

tel [2]. An important advantage of this structure is its oversized dimension compared to the laser wavelength. The structure consists of stacked elements of conic shape (see Ref. [3]). The eigenmodes of the structure were analytically derived in Reference [4]. Given the laser frequency in vacuum $\omega$, the electric field associated to the TM mode (no azimuthal dependence) is given by:

$$
\begin{aligned}
E_{z}(r, z, t) & =\hat{E} J_{0}\left(k_{r} r\right) e^{i\left(k_{z} z-\omega t\right)} \text { and } \\
E_{r}(r, z, t) & =\frac{k_{z}}{k} Z_{0} H_{\phi}(r, z, t) \\
& =-i \frac{k_{z}}{k_{r}} \hat{E} J_{1}\left(k_{r} r\right) e^{i\left(k_{z} z-\omega t\right)}
\end{aligned}
$$

where $\hat{E}$ is the axial peak electric field, $k=n \omega / c$ ( $n$ being the index of refraction of the medium), $k_{r}=\frac{p_{10}}{a[1+\eta(1+i) / M]}$ $\left(p_{01} \simeq 3.832\right)$ and $k_{z}=\sqrt{k^{2}-k_{r}^{2}} \simeq k-k_{r}^{2} /(2 k) . Z_{0}$ is the impedance for free space. Such a wave is supraluminous in vacuum: the phase velocity is

$$
v_{\phi} \doteq \frac{\omega}{\operatorname{Re}\left(k_{z}\right)} \simeq \frac{c}{n}\left[1+\frac{1}{2}\left(\frac{p_{10} \lambda}{2 \pi a}\right)\right],
$$

which is always larger than $c$ in vacuum $(n=1)$. This is a limitation when trying to accelerate non-ultra-relativistic incoming beams such as considered in the present paper. The laser phase velocity needs to be reduced by selecting a medium with the proper refractive index, e.g. immersing the interaction region in a gas.

\section{GENERATION OF TM ${ }_{01}^{*}$ MODE}

For efficient coupling of the incoming laser into the structure, the laser mode should be radially polarized. Such a laser has been developed at FNPL by the two of us (from University of Rochester) by employing a Mach-Zender interferometer [3]. A seed from the photocathode drive laser oscillator (a Nd:YLF oscillator) is regeneratively amplified and sent to the interferometer. The incoming $\mathrm{TM}_{01}$ mode is split $(50 \%)$ and one of the beams rotated by $90^{\circ}$ via a periscope and then recombined with the other beam with proper phase to yield the radially polarized $\mathrm{TM}_{01}^{*}$ mode. Figure 2 shows an example of measured intensity of the $\mathrm{TM}_{01}^{*}$ mode. To date the radially polarized mode has been generated but reproducibility and reliability still present challenges and further work is needed before confidently using this laser to drive our acceleration experiment. Furthermore amplification to high energies and compression in time of a radially polarized beam have not as yet been 

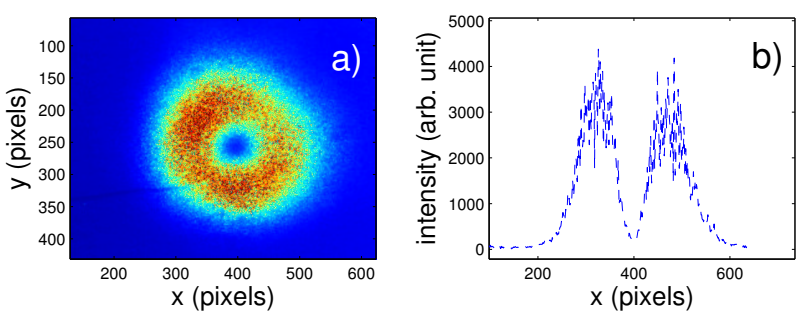

Figure 2: Measured transverse density a) and corresponding line profile $\mathbf{b}$ ) of the $\mathrm{TM}_{01}^{*}$ mode.

\begin{tabular}{ccc}
\hline \hline Parameter & Value & Unit \\
\hline charge & 100 & $\mathrm{pC}$ \\
total energy & 43 & $\mathrm{MeV}$ \\
transverse emittance & 0.7 & $\mathrm{~mm}-\mathrm{mrad}$ \\
bunch length & $0.5(1.7)$ & $\mathrm{mm}(\mathrm{ps})$ \\
momentum spread & 5.5 & $\mathrm{KeV}$ \\
\hline \hline
\end{tabular}

Table 1: FNPL nominal operating settings and simulated parameters for $100 \mathrm{pC}$ bunch.

demonstrated. For efficient coupling between the $\mathrm{TM}_{01}^{*}$ mode and the eigenvalue mode of the structure, we need to focus the mode to a waist $w \simeq 0.6 a$ where a is the iris diameter. Preliminary tests in propagating the laser beam in the structure indicate a transmission of $\sim 80 \%$ in a $25 \mathrm{~cm}$ long structure consisting of 250 conic stacked elements.

\section{EXPECTED PERFORMANCES AT FNPL}

The laser acceleration of electrons using the OILS concept is planned at FNPL, once the energy upgrade of the facility is completed (for a description see Reference [7]), at a beam energy $E>40 \mathrm{MeV}$. We assume for the subsequent calculations of the laser acceleration performance a $40 \mathrm{MeV} \mathrm{e} \mathrm{e}^{-}$beam. For the laser acceleration, we need, at the interaction location, an $\mathrm{e}^{-}$beam that can be focused to transverse spot sizes $\sigma_{x}=\sigma_{y} \simeq 200 \mu \mathrm{m}$ (rms). Such a small focus insures the 4- $\sigma$ beam is contained within the structure aperture (delimited by the iris radius $a=1 \mathrm{~mm}$ ). Therefore we must optimize the $\mathrm{e}^{-}$transport to produce a low emittance beam. Since the charge per bunch is not a limiting parameter, we optimized the accelerator at the charge $Q=100 \mathrm{pC}$, low enough to achieved transverse normalized emittance below $1 \mathrm{~mm}$-mrad and, in the meanwhile, large enough to allow the use of our diagnostics (optical transition radiation and scintillating YAG screens). The main parameters of the low charge settings for the injector are reported in Table 1. For our estimate of the laser acceleration we consider an OILS with active length $L=10 \mathrm{~cm}$ and iris radius $a=1 \mathrm{~mm}$, the anticipated peak field is $\hat{E}=9 \mathrm{MV} / \mathrm{m}$.

In order to have a net electrons energy change, the laser and $\mathrm{e}^{-}$beams must be synchronous, i.e. the phase velocity of the laser should match the $\mathrm{e}^{-}$beam velocity. Two causes may break the synchronous condition: (1) the incoming beam is non-ultra-relativistic, and (2) when the energy gain is too high there will be significant phase slippage between the laser and $\mathrm{e}^{-}$beams. To avoid these effects, the laser phase velocity is decreased by immersing the interaction region in a gas with index of refraction chosen to have:

$$
n-1=\frac{1}{2}\left[\left(\frac{p_{01} \lambda}{2 \pi a}\right)^{2}+\frac{1}{\gamma^{2}}\right] .
$$

From past experience [6] with $\mathrm{e}^{-}$beam having similar parameters, filling the interaction region with hydrogen would be the best alternative. For Hydrogen $\left(\mathrm{H}_{2}\right)$, the index of refraction, at a given wavelength $\lambda$, is parameterized in term of gas pressure and temperature via the relation:

$$
n-1=10^{-6} \frac{P}{760} \frac{273.15}{T}\left(21.113+\frac{12723.2}{111-\lambda^{2}}\right)
$$

with $P, T$ and $\lambda$ respectively given in units of Torr, Kelvin and microns. For the nominal energy $\gamma=78$ we estimate from Eq. 3 for the refractive index $n-1 \simeq 8.3 \times 10^{-5}$, corresponding to a pressure of $\mathrm{H}_{2}$ of 450 Torr (see Fig. 3).
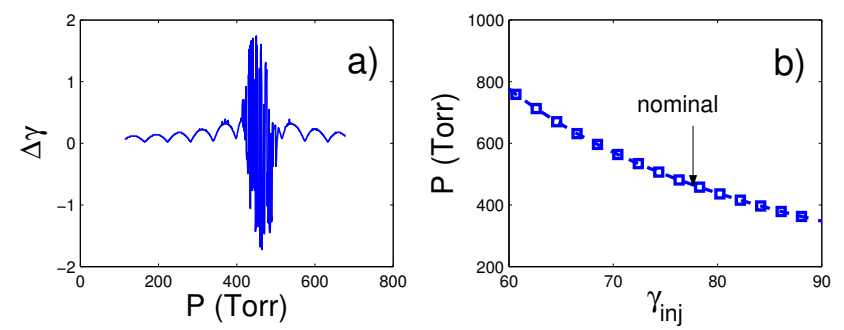

Figure 3: Sensitivity of energy gain on gas pressure (a) and required gas pressure for incoming energy (b) .

The two major effects that may degrade the expected signature of the laser $/ \mathrm{e}^{-}$interaction are (1) multiple scattering as the beam crosses the vacuum window and propagate in the gas and (2) the effect of wakefields associated to the beam Coulomb field interacting with the structure. The use of a gas at significant pressure (order of $1 \mathrm{~atm})$ to slow the phase velocity of the laser will deteriorate the $\mathrm{e}^{-}$beam via beam-gas scattering. The total emittance growth due to scattering is approximately $\Delta \tilde{\varepsilon}_{x} \simeq \sum_{i} 2 / 3(28 / \gamma)^{2}\left[L^{(i)} / X_{0}^{(i)}\right]$, where the summation is performed on the different constituents degrading the beam (two beryllium windows and the gas volume), $X_{0}$ and $L$ are the radiation length and interaction length. Our estimate results in a beam blow-up by a factor $\sim 2$ at the end of the structure. However extrapolation of Ref. [6] are more optimistic. Longitudinal wake-field produced will cause the bunch energy spread to growth, this effect is indeed small at $100 \mathrm{pC}$ for the considered bunch duration $\left(\sigma_{t} \simeq 2 \mathrm{ps}\right)$.

To estimate the performance of the laser acceleration experiment at FNPL, we use the tracking program ASTRA from DESY [5] . A new cavity element was introduced 
in the program based on the Eq. 1. The program then integrates the equation of motion taking into account space charge and the laser external fields. In the calculations reported here we do no include the beam-scattering due to the gas filling the interaction region and entrance/exit vacuum windows. We also assume the entire $\mathrm{e}^{-}$beam is interacting with the laser beam while in reality the laser beam may be shorter than the $\mathrm{e}^{-}$beam. The main signa-
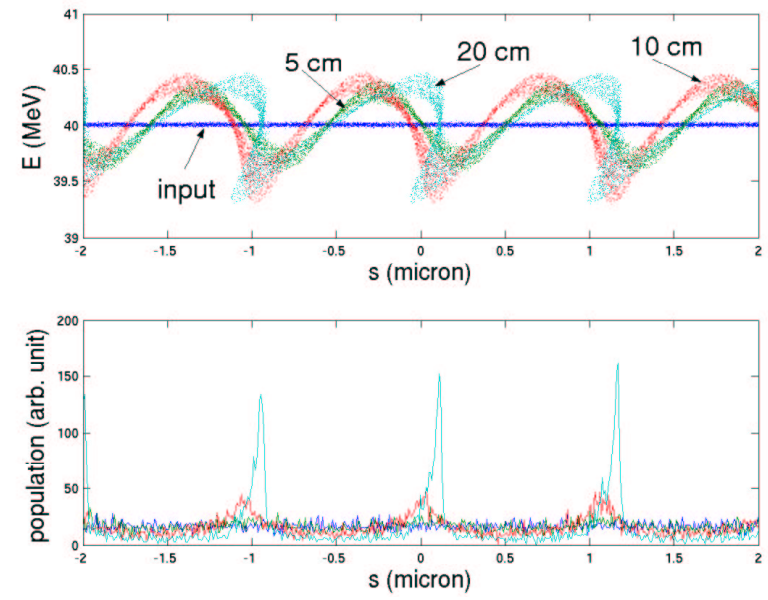

Figure 4: (top) longitudinal phase space upstream (input), and at different locations downstream of the OILS section entrance $(5,10$ and $20 \mathrm{~cm})$ along with the associated charge density (bottom) ( $s>0$ corresponds to the bunch head).

ture of the laser/e ${ }^{-}$interaction occurs on the $\mathrm{e}^{-}$longitudinal phase space. Because the laser wavelength is much shorter than the bunch length, an energy modulation is imparted onto the $\mathrm{e}^{-}$beam (see Fig. 4). The build-up of energy modulation and the associated density modulation is seen in Fig. 4. This results in a double-peaked energy spec-

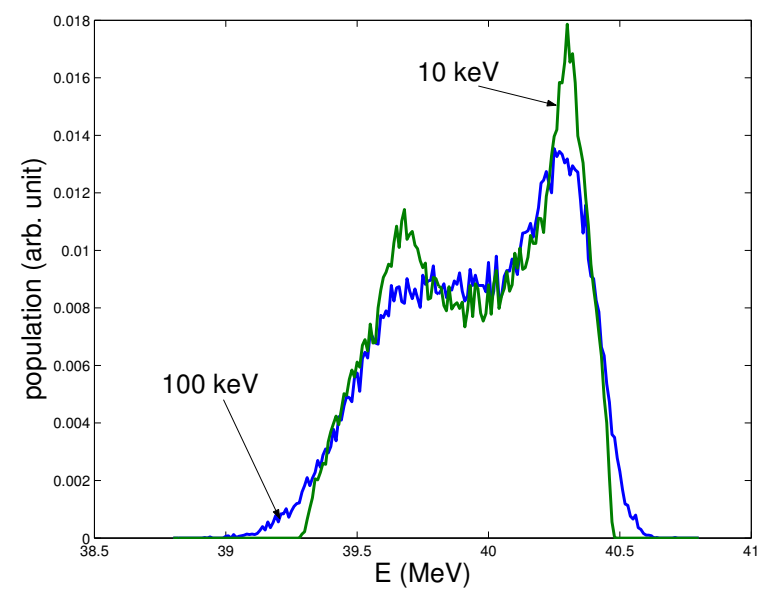

Figure 5: Sensitivity of the energy spectrum on the incoming $\mathrm{e}^{-}$beam energy spread $\left(\sigma_{x, y}=0.1 \mathrm{~mm}\right)$.

trum as shown in Fig. 5. The influence of incoming energy spread is not significant: even for large incoming energy spread (100 keV rms) the spectrum is not significantly al- tered. Because of the radial dependence of the longitudinal field we also investigate the evolution of energy spectrum for various $\mathrm{e}^{-}$beam sizes in the OILS (see Fig. 6). The results indicates it is necessary to keep the beam sizes small $\left(\sigma_{x, y}<200 \mu \mathrm{m}\right)$ in order to avoid smearing of the doublepeak structure. An advantage of accelerating non-ultrarelativistic $\mathrm{e}^{-}$beams is that the energy modulation eventually converts into a density modulation at the laser wavelength (micro-bunching). The maximum compression is achieved after a drift length $D=1 / \gamma^{2}(d \delta / d s)^{-1} \simeq 10 \mathrm{~cm}$ downstream of the OILS (where $d \delta / d s$ is the fractional energy chirp imparted by the laser). The micro-bunching
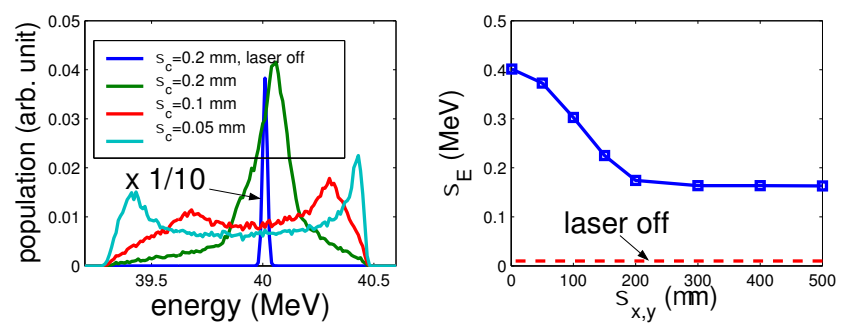

Figure 6: Simulated energy spectrum for various $\mathrm{e}^{-} 1$ beam sizes in the OILS structure (left) and corresponding energy spread (right). Note for $\sigma_{x, y}>0.2 \mathrm{~mm}$, the beam is not fully transmitted $\left(\sigma_{E}=10 \mathrm{keV}\right)$. (Note that the profile corresponding to the "laser off" case has been scaled by 1/10.)

could be used for other advanced accelerator physics applications (e.g. injection of pre-bunched beam in a plasmawakefield accelerator); it could also produce coherent radiation, e.g., for bunch length diagnostics.

\section{CONCLUSION}

We have analyzed the possibility of performing an inverse Cerenkov acceleration experiment at FNPL with a 40 $\mathrm{MeV}$ electron beam. A clear signature of laser/electron interaction should be obtained in this experiment for a large range of incoming electron beam parameters. The $\mathrm{TM}_{01}^{*}$ mode laser has been developed and the experiment will be performed after the FNPL upgrade [7].

\section{REFERENCES}

[1] J.D. Lawson, IEEE Trans. Nucl. Sci. NS-26, p, 4217 (1979); P.M. Woodward, J. IEEE 93, p. 1554 (1947).

[2] R. Pantel, Nucl. Instr. Meth. A 393, p. 1 (1997)

[3] R. Tikhoplav et al. proc. of EPAC 2002, p. 984 (2002)

[4] M. Xie, Berkeley reports LBNL-40558 (1997) and LBNL42055 (1998)

[5] K. Flöttemann, Astra user manual

[6] A. S. Fisher et al., Nucl. Instr. Meth. A 272, p. 89 (1988)

[7] P. Piot et al., this conference 